\title{
Multidimensional Optimization-Based Heuristics Applied to Wireless Communication Systems
}

\author{
Fernando Ciriaco, Taufik Abrão and \\ Paul Jean E. Jeszensky \\ Additional information is available at the end of the chapter \\ http://dx.doi.org/10.5772/54152
}

\section{Introduction}

In the last two decades, the mobile communications technologies and the Internet have grown almost exponentially, reaching a significant numbers of subscribers around the world. The mobile cellular service got a very large growth of users along with the increase of mobile data services. On the other hand, the Internet provides a great opportunity for users to access the information via fixed and/or wireless networks.

In this scenario, stands out the spread spectrum communication techniques that until the mid-80 were restricted to military applications and is currently in a final technological consolidation phase through the cellular mobile communication systems of third and fourth generations used throughout the world [1].

Such multiple access-based systems use a matched filter bank to detect the interest signal, being however unable to recover the signal in an optimal way, regardless is affected by additive white Gaussian noise (AWGN), flat fading or selective fading channels, since the direct sequence code division multiple access (DS/CDMA) signal is corrupted by multiple access interference (MAI) and severely affected by the near-far effect, resulting in a system whose capacity may remain remarkably below the channel capacity [2] if specific techniques are not introduced to mitigate these effects, such as multiuser detection (MuD) [3], diversity exploration $[4,5]$ and so forth.

Thus, one of the biggest challenges in the multiuser communication systems development is the interference mitigation. This challenge becomes obvious to the modern and current wireless networks like cellular networks, wireless local area network (WLAN) and wireless metropolitan area network (WMAN), due to the high spectral efficiency need, requiring advanced techniques for frequency reuse and interference mitigation.

The third and fourth generations of cellular mobile systems and wireless networks were designed to support many services through the use of multirate transmission schemes, different quality of service (QoS) requirements and multidimensional diversity (time, 
frequency and space). Thus, modern systems must accept users transmitting simultaneously in different rates in asymmetric traffic channels (uplink and downlink may be required to work at different rates), and also ensure the minimum specifications of QoS for each offered service.

Hence, current industry standards for wireless networks use a combination of the following techniques to improve the frequency spectrum efficiency: multicarrier, spread spectrum, multiple antennas, spatial multiplexing and coding, reinforcing researches in order to improve the capacity of these systems, considering efficient transmission schemes, multiple diversity combination, multiuser detection methods, among others.

\subsection{Multiuser detection}

One way to reduce substantially the interference and increase spread spectrum system capacity consists in modifying the detection strategy, using the information of other interfering users signals for detection process of interest user information. This strategy is called multiuser detection $(\mathrm{MuD})[3,6,7]$.

In $\mathrm{MuD}$ strategy, active user information in the system are used together in order to better detect each individual user, increasing the system performance and/or capacity.

From the 1986 pioneering Verdu's work [3, 6] on optimum multiuser detector (OMuD) to a wide variety of multiuser detectors aiming to improve the performance obtained with the conventional detector in multiple access systems, a remarkable advance in the field has been achieved in the last twenty years. However, given the exponential complexity of the optimum detector, the research efforts has been focused on the development of sub-optimal or near-optimal multiuser detectors with lower complexity.

Alternatives to $\mathrm{OMuD}$ include the classical linear multiuser detectors such as Decorrelator [3] and the minimum mean square error (MMSE) [8], the nonlinear MuDs, such as interference cancellation (IC) $[9,10]$ and zero forcing decision feedback (ZF-DFE) detector [11], and heuristics-based multiuser detectors $[12,13]$.

However, both classical linear MMSE and Decorrelator multiuser detector algorithms presents two drawbacks; a) for practical system scenarios, both MuD result in performance degradation regarding $\mathrm{OMuD}$; $\mathrm{b}$ ) they need to perform a correlation matrix inversion, which implies in a high complexity for practical wireless systems with a high number of active users and/or systems with real-time detection in with the active number of users randomly and quickly changes along the time.

The operation principle for the non-linear classical IC and ZF-DF multiuser detectors is the reconstruction of MAI estimates, followed by cancellation (subtraction) for the interest user signal. The operations of MAI reconstruction and cancellation can be repeated in a multistage structure, resulting in more reliable signals canceling each new stage when estimates can be obtained with relative accuracy. The complexity of these detectors increases with the number of necessary stages for demodulation and after a certain number of stages there is no significant performance gain due to the propagation of interference estimation error. This limits the performance of these algorithms. Although the advantage of lower complexity regarding the MMSE and Decorrelator, performance achieved by the non-linear subtractive $\mathrm{MuD}$ detectors remain below the MMSE detector for almost all practical interest scenarios. 


\subsection{Heuristics applied to communication systems}

In the last decade, the literature has been collecting sub-optimal solutions proposals based on iterative algorithms and heuristics, particularly evolutionary and local search, applied to inherent multiple access communication systems problems, among which we could cite the following heuristic solutions: optimal multiuser detection [12-19]; spreading sequences selection [20, 21]; parameter estimation, particularly the channel coefficients estimation, delay and power users [19, 22, 23]; power control problem [24, 25]; and resource allocation optimization [26-28].

However, in recent years, the multiuser detection optimization problem in a single DS/CDMA system have been changed for others more complex applications, such as systems with multiple transmit and receive antennas, multirate coded systems with different quality-of-services, and multicarrier CDMA systems.

Differently from the most results reported in the literature, this chapter considers a multidimensional approach which aims to recover optimally (or very close to the optimal point) all the information of all users within the same processing window, considering multipath channels with different power-delay profiles, data rates, time or space-time coding, multiple antenna and multicarrier. In dealing with this sort of system, it will possible to provide various high-rate user's services including voice, data and video under a scenario with growing scarceness of spectrum and energy.

Moreover, to establish quality criteria that meet the acceptance requirements of the scientific community and telecommunications industry standards, this work analyzes convergence and performance aspects of a wide representative heuristic algorithms, considering metrics such as stability, capacity, control and implementation aspects, as well as the algorithm complexity in relation to conventional topologies.

However, aiming at the multidimensional optimization analysis for high performance systems, various techniques based on heuristic algorithms are deployed in this work. Heuristic algorithms have been applied in several optimization problems, showing excellent results in large combination problems for practical cases. Still, there is an inherent difficulty in selecting and setting up the algorithm steps, since correct choices will result in good performances, and contrary, a poorly calibrated parameters may result in a disastrous performance.

Therefore, the manipulation of several variables associated with each heuristic algorithm requires knowledge of the problem to be optimized, experience and keen perception of the algorithm behavior when selecting the parameters. Often, the parameters used are appropriate only for very restrictive settings, and frequently there are no consensus on the (sub-)optimal input parameters to be adopted or even the more conducive internal strategies to adjust those input parameters. Thus, parameters are chosen by past accumulated experience in dealing with other optimization problems or even through non-exhaustive trial tests. This scenario has resulted in a somewhat distrust level of such alternatives application in optimization problems that commonly arise in communications systems.

Thus, the motivation in pursuit of heuristic algorithms to ensure optimal performance is the core of this chapter. To do so, it will be analyzed in a systematic way the main meta-heuristic and hyper-heuristic algorithms deployed in wireless systems, which may be mentioned the genetic algorithm, evolutionary programming, local search ( $k$-optimum), 
simulated annealing, heuristic algorithm based on Tabu list and a hyper-heuristic-basis selection.

\section{System characteristics}

Considering the provision of various services with high quality, we opted a transmission/reception scheme that adds several dimensions in order to explore diversity. Figure 1 shows the transmitter and receiver topologies deployed in this work. Hence, lets consider the $k$-th user transmitter and noting that the channel coding stage is necessary to correct the received signal in the presence of errors through the use of redundancy (code diversity). The multirate modulation block aims to ensure the provision of various services to users at different data rates, ensuring the possibility of optimum resource management strategies. The time-spreading code guarantees a rejection level of multiple access interference, and the identification of each DS/CDMA user as well, acting as a kind of time-diversity. The frequency spreading block implements frequency-diversity through the information transmission on different sub-carriers. Finally, the multiple-input-multiple-output (MIMO) antennas block deploys techniques that provide spatial-diversity, either through simple arrangements with various antennas or even by space-time block code (STBC) or trellis code.

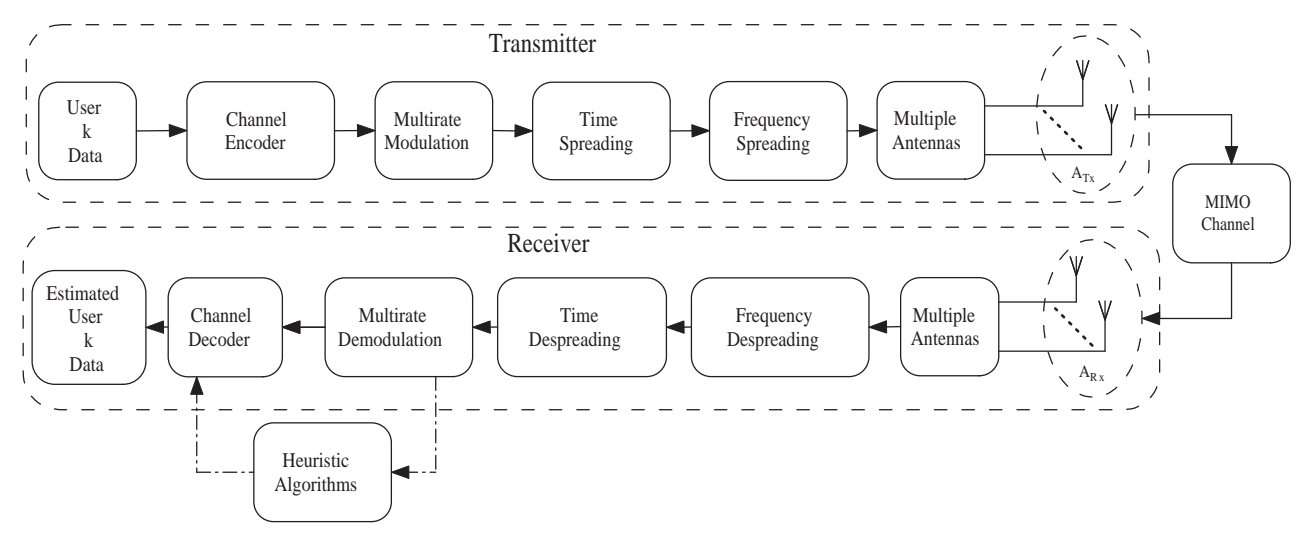

Figure 1. Communication system overview with use of space, time, frequency and coding diversities.

The transmitted signal of the $k$-th user propagates through a channel whose model includes attenuation of small and large scale, i.e., path loss, shadowing and multipath effects.

The $k$-th user signals at the receiver input are demodulated via an antenna array in order to exploit spatial diversity. Structures can be used with several receiving antennas physically separated by a sufficient distance to avoid overlapping signals and block-basis or trellis-basis signal processing techniques. Subsequently, the signals are despread in frequency and time ensuring the channel rejection and multiple access interference rejection, respectively. At this point, it is evident the frequency- and time-diversity exploitation. Thus, the demodulated signals are reassembled considering the $k$-th user transmission rates. This receptor is known as Rake receiver. Finally, the signals are decoded by means of particular techniques, resulting in a type of diversity code. 


\subsection{Received signal in multipath MIMO channels}

Considering the reverse link and assuming a set of bits transmitted (frame) consisting of $I$ bit for each multirate user, the resulting signal propagates through $G$ independent Rayleigh fading paths. Thus, the equivalent baseband received signal (assuming ideal low-pass filter) in one of the antennas is:

$$
\begin{gathered}
r_{n_{R x}}(t)=\sum_{i=0}^{I-1} \sum_{k=1}^{K^{(g)}} \sum_{g=1}^{G}\left[\sum_{m=1}^{M} \sum_{j=1}^{m^{(g)}} A_{k, g}^{\prime} \mathbf{x}_{k(g)}^{(i)}[j] s_{C k}^{(g)}(t-j T) s_{k}^{(g)}\left(t-\tau_{k, \ell}^{(g)}-i T\right) .\right. \\
\left.\cdot s_{F k, m}^{(g)} \cos \left(2 \pi f_{m} t+\phi_{k, m}^{(g)}\right)\right] * h_{k, g}^{(i)}(t)+\eta(t)
\end{gathered}
$$

where $K^{(g)}$ is the number of physical users belonging to $g$-th multirate group being $K=K^{(1)}+K^{(2)}+\ldots+K^{(g)}+\ldots+K^{(G)}$ the total number of active users in the physical system, divided into $g$ user groups of same rate, $t \in[0, T], M$ represents the number of subcarriers, the amplitude $A_{k, g^{\prime}}$ is the amplitude of the received $k$-th user of $g$-th multirate group, including the effects of path loss and shadowing channel, and assumed constant over the $I$ bits transmitted base rate, $\mathbf{x}_{k(g)}^{(i)}[j] \in\{ \pm 1\}$ is the symbol of coded information passed to the $i$ th symbol interval; $s_{C k}, s_{k}$ and $s_{F k, m}$ represent the sequences of channeling, time and frequency spread, respectively, $\tau_{k, \ell}^{(g)}$ is the random delay, $\phi_{k, m}^{(g)}$ corresponds to the initial $k$-th user; $f_{m}$ represents the respective subcarriers frequencies; $h_{k, g}$ is the impulse response of the channel and the term $\eta(t)$ is the AWGN with bilateral power spectral density equal to $N_{0} / 2$.

The $k$-th user delay of $g$-th multirate group takes into account the nature of the asynchronous transmission, $d_{k}^{(g)}$, as well as the propagation delay, $\Delta_{k, \ell}^{(g)}$ for $k$-th user, $\ell$-th path, $g$-th multirate group, resulting in:

$$
\tau_{k, \ell}^{(g)}=\Delta_{k, \ell}^{(g)}+d_{k}^{(g)}
$$

The channel impulse response to the $k$-th user of $g$-th multirate group in the range of $i$-th bit can be written as:

$$
h_{k, g}^{(i)}(t)=\sum_{\ell=1}^{L} c_{k, \ell, g}^{(i)} \delta\left(t-\Delta_{k, \ell}^{(g)}-i T\right)
$$

where $c_{k, \ell, g}^{(i)}=\beta_{k, \ell, g}^{(i)} e^{j \phi_{k, \ell, g}^{(i)}}$ indicates the complex channel coefficient for the $k$-th user of $g$-th multirate group, $\ell$-th path and $\delta(t)$ is the unit impulse function. It is assumed that the phase of $c_{k, \ell, g}^{(i)}$ have an uniform distribution $\phi_{k, \ell, g}^{(i)} \in[0,2 \pi)$ and the module channel $\beta_{k, \ell, g}^{(i)}$ represents the small-scale fading envelope with a Rayleigh distribution.

Additionally, we considered normalized channel gain for all users, i.e., $\mathbb{E}\left[\sum_{\ell=1}^{L}\left|c_{k, \ell, g}\right|^{2}\right]=1$ for $\forall k, g, i$. 
Therefore, we can rewrite the received signal in each $A_{R x}$ antennas replacing the eq.(3) into eq. (1), resulting in:

$$
\begin{aligned}
r_{n_{R x}}(t)= & \sum_{i=0}^{I-1} \sum_{k=1}^{K^{(g)}} \sum_{g=1}^{G} \sum_{m=1}^{M} \sum_{j=1}^{m^{(g)}} \sum_{\ell=1}^{L} A_{k, g, \ell}^{\prime} \mathbf{x}_{k(g)}^{(i)}[j] s_{C k}^{(g)}(t-j T) s_{k}^{(g)}\left(t-\tau_{k, \ell}^{(g)}-i T\right) \\
& \cdot s_{F k, m}^{(g)} \cos \left(2 \pi f_{m} t+\phi_{k, m}^{(g)}\right) c_{k, \ell, g}^{(i)} \delta\left(t-\Delta_{k, \ell}^{(g)}-i T\right)+\eta_{n_{R x}}(t)
\end{aligned}
$$

For simplicity and without generality loss, we consider ordered random delays, i.e.:

$$
0=\tau_{1,1}^{(1)} \leq \tau_{1,2}^{(1)} \leq \cdots \tau_{1, L}^{(1)} \leq \tau_{2,1}^{(1)} \leq \cdots \leq \tau_{K^{(1)}, L}^{(1)} \leq \cdots \tau_{K^{(G)}, L}^{(G)}<T
$$

\subsection{Conventional SIMO detection systems}

Considering the system with only one transmission antenna, one can rewrite eq. considering just the $n_{R x}$-th receiving antenna and $m$-th subcarrier as:

$$
\begin{array}{r}
r_{n_{R x}, m}(t)=\sum_{i=0}^{I-1} \sum_{k=1}^{K^{(g)}} \sum_{g=1}^{G} \sum_{j=1}^{m^{(g)}} \sum_{\ell=1}^{L} A_{k, g, \ell} \mathbf{x}_{k(g)}^{(i)}[j] s_{C k}^{(g)}(t-j T) s_{k}^{(g)}\left(t-\tau_{k, \ell}^{(g)} i T\right) s_{F k, m}^{(g)} . \\
\cdot \beta_{k, \ell, g, m}^{(i)} e^{j\left(\omega_{m} t+\varphi_{k, \ell, g, m}^{(i)}\right)}+\eta_{n_{R x}}(t)
\end{array}
$$

where $\eta_{q}(t)$ corresponds to the Additive White Gaussian Noise (AWGN) for $n_{R x}$-th receiving antenna.

For multipath fading channels, multirate and multicarrier scheme, the receiver for each subcarrier demodulation use the Rake receiver consisting of a bank of $K D$ matched filters to the multirate physical users spread sequences with path diversity $\operatorname{order}^{1} D \leq L$, followed by the second despreading (channeling) aiming recovering $m^{(g)}$ simultaneously transmitted bits in parallel channels. To be able achieve a perfect synchronism (maximum auto-correlation) of spread sequence at the receiver must use delay accurate estimates for the $\ell$-th path of the $k$-th user of $g$-th multirate group, $\hat{\tau}_{k, \ell}^{(g)}$. Performance is degraded proportionally when there are errors in the delays estimates.

Thus, the $m^{(g)}$ matched filter outputs for the $k$-th physical user, $g$-th multirate group and corresponding to $\ell$-th multipath component, $m$-th subcarrier and $n_{R x}$-th antenna, sampled at the end of basic information period $T$ of $i$-th interval symbol can be expressed as:

$$
\begin{aligned}
y_{k, \ell, g, n_{R x}, m}^{(i)}[j] & =\frac{1}{\sqrt{N_{C}}} \int_{0}^{T} r_{n_{R x}, m}(t) s_{k}^{(g)}\left(t-\tau_{k, \ell}^{(g)}-i T\right) s_{C k}^{(g)}(t-j T) s_{F k, m}^{(g)} e^{\left(-j \omega_{m} t\right)} d t \\
& =\underbrace{A_{k, g}^{\prime} T c_{k, \ell, g, m}^{(i)} \mathbf{x}_{k(g)}^{(i)}[j]}_{(I)}+\underbrace{S I_{k, \ell, g, n_{R x}, m}^{(i)}}_{(I I)}+\underbrace{M A I_{k, \ell, g, n_{R x}, m}^{(i)}}_{(I I I)}+\underbrace{n_{k, \ell, g, n_{R x}, m}^{(i)}}_{(I V)}
\end{aligned}
$$

\footnotetext{
${ }^{1}$ If $D<L$, in each Rake receiver the matched filters to spread sequences are synchronized to $D$ energy major paths.
} 
where $j=1: m^{(g)}$. The first term corresponds to the desired signal, the second term to the self-interference (SI), the third to the MAI on the $\ell$-th multipath component of the $k$-th user of $g$-th multirate group, $m$-th subcarrier and $n_{R x}$-th antenna, as well the last term corresponds to the filtered AWGN.

In this case, the Rake receiver combines the outputs of the matched filters bank available for each user (fingers) ${ }^{2}$ and weighted by the respective channel gains [29]. The Maximal Ratio Combiner (MRC) combines the signals from the $D$ correlators in coherent way:

$$
\hat{\mathbf{y}}_{k, g}^{(i)}=\sum_{\ell=1}^{D} \sum_{n_{R x}=1}^{A_{R x}} \sum_{m=1}^{M} \Re\left\{\mathbf{y}_{k, \ell, g, n_{R x}, m}^{(i)} \hat{\beta}_{k, \ell, g, n_{R x}, m}^{(i)} e^{-j \hat{\phi}_{k, \ell, g, n_{R x}, m}^{(i)}}\right\}
$$

where $\Re\{$.$\} is the real part operator, \hat{\beta}_{k, \ell, g, n_{R x}, m}^{(i)}$ and $\hat{\phi}_{k, \ell, g, n_{R x}, m}^{(i)}$ are the magnitude and phase estimates of the channel coefficients, respectively, for the $i$-th processing interval for the $k$-th user, $\ell$-th path, $g$-th multirate group, $n_{R x}$-th antenna and $m$-th subcarrier. Again, the performance is degraded proportionally when there are errors in the channel estimates.

Finally, the estimates for the $m^{(g)}$ information symbols of $k$-th user of $g$-th multirate group are obtained through an abrupt decision rule:

$$
\hat{\mathbf{x}}_{k, g}^{(i)}=\operatorname{sgn}\left(\hat{\mathbf{y}}_{k, g}^{(i)}\right)
$$

Therefore, the estimated symbol frame for all users in the range of $i$-th bit with $D K_{v} \times 1$ dimension is given by:

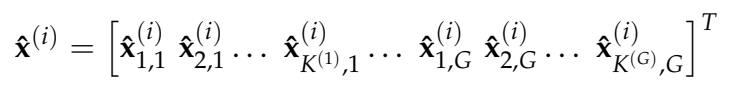

The performance obtained with the MRC Rake receiver will be deteriorated considerably when the number of users sharing the same channel grow ${ }^{3}$ and/or when the interfering users power increase.

\subsection{Optimum multiuser detector}

The best performance among the multiuser detectors is achieved with $\mathrm{OMuD}$, where the goal is to maximize the maximum likelihood function [3]. Given the conditional probability:

$$
P_{r}\left(\mathbf{y}^{(i)} \mid \hat{\mathbf{x}}^{(i)}, i \in[0, I-1]\right)=e^{\left\{-\frac{1}{2 \sigma^{2}} \int_{i=0}^{I-1}\left[\mathbf{y}^{(i)}-\mathbf{S}_{t}\left(\hat{\mathbf{x}}^{(i)}\right)\right]^{2} d t\right\}}
$$

\footnotetext{
${ }^{2}$ In addition to multipath effects, multiple subcarriers and multiple receiving antennas.

${ }^{3}$ Increasing the MAI, third term of eq. (7).
} 
where the total received signal, reconstructed from the estimated parameters and known at the receiver is:

$$
\begin{array}{r}
\mathbf{S}_{t}\left(\hat{\mathbf{b}}^{(i)}\right)=\sum_{k=1}^{K^{(g)}} \sum_{g=1}^{G} \sum_{m=1}^{M} \sum_{j=1}^{m^{(g)}} \sum_{n_{R x}=1}^{A_{R x}} \sum_{\ell=1}^{L} A_{k, g, \ell, n_{R x}}^{\prime} \hat{\mathbf{x}}_{k, g}^{(i)} \mathbf{s}_{C k}^{(g)}(t-j T) s_{k}^{(g)}\left(t-\tau_{k, \ell, n_{R x}}^{(g)}-i T\right) \\
\cdot s_{F k, m}^{(g)} \hat{\beta}_{k, \ell, g, n_{R x}}^{(i)} e^{j\left(\omega_{m} t+\hat{\phi}_{k, \ell, g, n_{R x}}^{(i)}\right)}
\end{array}
$$

In this context, the maximum likelihood vector that must be found by $\mathrm{OMuD}$ has $D K_{v} \times 1$ dimension, and by:

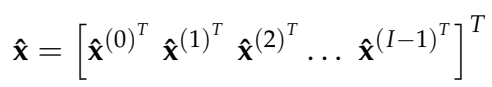

Note that the minimum square difference exists in eq. (11) ensures the maximization of the maximum likelihood function. Expanding the quadratic difference in eq. (11), based on the output of the matched filter, vector $\mathbf{y}^{(i)}$, find the maximum likelihood vector $\hat{\mathbf{x}}$ is equivalent to selecting the bit vector $\mathcal{B}$, with same size, which maximizes the Log Likelihood Function (LLF) [3]:

$$
\Omega(\mathcal{B})=2 \sum_{n_{R x}=1}^{A_{R x}} \Re\left\{\mathcal{B}^{T} \mathcal{C}^{H} \mathcal{A} \mathcal{Y}\right\}-\mathcal{B}^{T} \mathcal{C} \mathcal{A} \mathbf{R} \mathcal{A C}^{H} \mathcal{B}
$$

where each matrix must be determined for each receiving antenna and $(\cdot)^{H}$ refers to the Hermitian transpose operator.

The diagonal channel coefficients and amplitudes matrices ${ }^{4}$ for $n_{R x}$-th receiving antenna of $D K_{v} I$ dimension are defined, respectively, by:

$$
\begin{aligned}
& \mathcal{C}=\operatorname{diag}\left[\mathbf{C}^{(0)} \mathbf{C}^{(1)} \mathbf{C}^{(2)} \ldots \mathbf{C}^{(I-1)}\right] \\
& \mathcal{A}=\operatorname{diag}\left[\begin{array}{llll}
\mathbf{A}^{(0)} & \mathbf{A}^{(1)} & \mathbf{A}^{(2)} \ldots \mathbf{A}^{(I-1)}
\end{array}\right]
\end{aligned}
$$

The output vector of the matched filter (MFB), composed by $I$ vectors $\mathbf{y}^{(i)}$ with dimension $D K_{v} I \times 1$ is given by:

$$
\mathcal{Y}=\left[\mathbf{y}^{(0)} \mathbf{y}^{(1)} \mathbf{y}^{(2)} \ldots \mathbf{y}^{(I-1)}\right]^{T}
$$

In general, the MFB output vector $\mathcal{Y}$ is deployed as initial guess in the LLF cost function, eq. (14) when a heuristic multiuser detection is performed. The general rule should be ensure the maximization of the cost function, considering the same $\mathcal{Y}$ for all receiving antennas.

\footnotetext{
${ }^{4}$ To simplify the notation, hereafter we have omitted the matrix index $n_{R x}$.
} 
Finally, the block Toeplitz tridiagonal correlation matrix $\mathbf{R}$, dimension $D \mathcal{K}_{v} I \times D \mathcal{K}_{v} I$, is defined as [3]:

$$
\mathbf{R}=\left[\begin{array}{cccccc}
\mathbf{R}[0] & \mathbf{R}^{T}[1] & \mathbf{0} & \cdots & \mathbf{0} & \mathbf{0} \\
\mathbf{R}[1] & \mathbf{R}[0] & \mathbf{R}^{T}[1] & \cdots & \mathbf{0} & \mathbf{0} \\
\mathbf{0} & \mathbf{R}[1] & \mathbf{R}[0] & \ddots & \mathbf{0} & \mathbf{0} \\
\vdots & \vdots & \ddots & \ddots & \ddots & \vdots \\
\vdots & \vdots & \vdots & \ddots & \mathbf{R}[0] & \mathbf{R}^{T}[1] \\
\mathbf{0} & \mathbf{0} & \mathbf{0} & \cdots & \mathbf{R}[1] & \mathbf{R}[0]
\end{array}\right]
$$

Therefore, the complete frame for the $I$ estimated symbols from all $K_{v}$ users can be obtained by optimizing eq. (14), resulting in:

$$
\widehat{\mathbf{x}}=\arg \left\{\max _{\mathcal{B} \in\{ \pm 1\}^{\mathcal{K}_{v} I}}[\Omega(\mathcal{B})]\right\}
$$

The $\mathrm{OMuD}$ consists in finding the best data symbols vector in a set with all the possibilities, i.e., it is a NP-complete combination problem [30], which the traditional algorithms are inefficient. Most of these result in exponential complexity growth when one or more of the following factors: number of users, frame, number of receiver antennas, number of paths, number of subcarriers, among others increase.

Therefore, the use of heuristic methods for this class of problems shows up attractive, since it is possible to obtain optimal solutions (or near-optimal) using reduced search spaces. Thus, the proposed strategy in this Chapter aiming for maximize the LLF by testing different candidates symbols vectors at each new iteration/generation of heuristic algorithms. Such attempts seek to maximize the system average performance, approaching or even equaling that obtained by $\mathrm{OMuD}$, but with remarkable reduction in the computational complexity.

\section{Heuristic algorithms}

This section presents a brief review of heuristic algorithms, specifically local and evolutionary search, describing variants and required parameters. Such variants include encoding (mapping) problem, initialization algorithms step (parameters choice), cost function evaluation, search space scanning step, and replacement candidates step. For the analysis, 1-opt and k-opt local search, simulated annealing, short-term and reactive Tabu search, genetic, as well evolutionary programming algorithms have been considered in this work.

\subsection{Encoding problem}

The encoding for MuD problem is inherently binary, because the data vector is naturally binary. Therefore, following the Keep it Simple (KIS) principle as much as possible, it is not necessary to perform an encoding (mapping) of candidate solutions differently to binary form. Thus, these candidates vectors will be directly represented by the information bits that will be tested by the cost function, considering only polarized binary encoding, i.e., for each candidate position of the vector $\mathcal{Y}$ is able to assume just only one value in the set $\{ \pm 1\}$. 


\subsection{Search space definition}

After the encoding step, we must define the problem search space, in which case the MuD problem is characterized by all possible combinations bits that users can transmit. In this case, for $K_{v}$ virtual users transmiting $I$ bits through a multipath channel with $L$ paths and $D$ processing signal branches at the receiver, the total search universe, considering optimizing the output of matched filter and signals combination from multirate users, is a binary set of dimension:

$$
\Theta\left(K_{v}, I, D\right)=2^{D K_{v} I}, \quad \text { with } \quad 1 \leq D \leq L .
$$

It is evident that for the proposed MuD problem, a total search universe should result smaller than $2^{D K_{v} I}$, since each transmitted bit must be detected in a way that results in a same estimated bit value for all $D$ processing branches, namely:

$$
\hat{b}_{k, 1, g}^{(i)}=\hat{b}_{k, 2, g}^{(i)}=\ldots=\hat{b}_{k, D, g}^{(i)} \in\{+1,-1\}
$$

This implies that the search universe covered by the heuristic MuD algorithm should be independent of the number of paths, resulting in:

$$
\Theta\left(K_{v}, I\right)=2^{K_{v} I}
$$

As a result, the universe of possible solutions is then formed by all vectors candidates that satisfy (21).

The other possibilities belong to the so-called forbidden universe, composing the non-tested candidates set into a heuristic methodology. This guarantees the final solution quality in $\mathrm{MuD}$ problem with multipath diversity, because it enable a correct estimate for all paths of the same transmitted bit could be made.

\subsection{Evolutionary Programming (EP) algorithms}

The evolutionary heuristic algorithms methods are non-deterministic search mechanisms based on natural selection and evolution mechanisms from Darwin's theory [31] $]^{5}$ This theory explains the life history by the physical processes action and genetic operators in populations or species. These processes are known for reproduction, disturbance, competition and selection.

Considering the computational implementation aspects, the parameters and strategies such as population size, mating pool size, selection strategy, crossover type and rate, mutation type and rate and replacement strategy should be chosen carefully by the user for each class of optimization problem, allowing numerous plausible combinations [33-35].

\footnotetext{
${ }^{5}$ Because [31] is a rare and difficult access reference, we consider newer editions of the Darwin's work, for instance [32].
} 


\subsection{Local Search (LS) algorithms}

The Local Search (LS) strategy is based on the better established existing principle for combinatorial optimization methods: trial and error. This is a natural and simple idea, but in fact, surprised by the success degree that this method has the most varied types of combinatorial problems.

The only parameters to be selected corresponds to the search starting point and the neighborhood size. The choice of starting point is usually done by intuition, because very few problems have a guide or direction.

For neighborhood definition, it should be pointed out that small neighborhood set leads in a low complexity algorithm, since the search space consists of few alternatives. On the other hand, the reduced size of the neighborhood set may not provide good solutions due to local minimum or maximum in this reduced region. Large neighborhood sets, on the other hand, provide good solutions but bring much greater complexity, since these sets may result in search space as large as brute force methods [36].

\subsection{Simulated Annealing (SA) algorithms}

The simulated annealing (SA) algorithm proposed by Kirkpatrick [37] was inspired by the annealing process of physical systems. This was based on the algorithm originally proposed by Metropolis [38] as a strategy for determining equilibrium states (or configurations) of a collection of atoms at a given temperature. The basic idea comes from the statistical thermodynamics, which is a physics branch responsible for theoretical predictions about the behavior of macroscopic systems based on the laws that govern their atoms. Using analogies, the SA algorithm was proposed based on the similarity between the annealing procedure implemented by the Metropolis algorithm and combinatorial optimization processes.

Thus, the concept of the SA algorithm is associated with the principle of thermodynamics, in which a solid heated to a very high temperature and then cooled gradually tends to solidify to form a homogeneous structure with lowest energy [37, 39].

This way, the SA algorithm must be started with one strategy and three parameters: initial temperature, $T(0)$, cooling step, $\epsilon$, size range (plateau) $L_{S A}$, and acceptance probability equation.

\subsection{Tabu search}

Tabu search algorithm was originally proposed in 1977 with the pioneering Glover's work [40] and later described in its current form in 1986 [41], being used in various knowledge areas and fields.

The short-term Tabu search (STTS) algorithm is based on the deterministic mode of memory operation. The memory is implemented by recording characteristics of displacement of previously visited solutions. This is described by the Tabu list, which is formed by the recent past search, being called the short-term memory effect. These displacement characteristics are prohibited by Tabu list for a number of iterations. This helps prevent returns to local solutions, promoting diversification in the search. 
The reactive Tabu search (RTS) version combines the short-term memory effect with another memory effect to avoid the local maximum returns and ensure efficient search. This effect is known as long-term memory, which alternates between intensification and diversification phases, adapting the prohibition period during the search, provide that the prohibition period takes different values for each iteration [42].

\subsection{Hyperheuristic strategies}

Over the past 50 years, the well-known meta-heuristic algorithms have been used as optimization tool for a wide range of optimization problems. The ability of the meta-heuristic algorithms to avoid local optimum-solutions offer us the ability to adapt this class of optimization strategy to solve various problems with the robustness and easiness of implementation, contributing to various optimization fields, mainly in those problems where deterministic or traditional optimization methods become inefficient or highly complex.

However, it is not easy or even possible to predict which of the many existing heuristic algorithms is the best choice for a specific optimization problem and be able to produce the same result given the same input parameters. The difficulty of choosing is associated to the performance unpredictability, which constitutes the major factor limiting their use by the scientific community and industry.

Furthermore, for a large optimization problems variety, the input parameter values should be controlled as the search evolves. In this context, recently, the idea of working with a higher level of automation in a heuristic design have been resulted in the development of the so-called hyper-heuristic (HH) strategy [43, 44].

Thus, the $\mathrm{HH}$ algorithms consist in applying a high-level methodology to control the selection or generation of generic search strategies using a specific number of different low-level heuristics.

It is worth noting that meta-heuristics are quality techniques to solve complex optimization problems, but efficient implementations of these methods usually require many specific knowledge about the problem being treated. Thus, the $\mathrm{HH}$ methodologies have been proposed aiming to build robust optimization algorithms, allowing the use of meta-heuristics methods with minor adaptations.

For HHs based on heuristics selection, one should choose the suitable number of iterations for the $\mathrm{HH}$, the selection strategy, as well as the acceptance strategy.

\section{Performance metrics for heuristic algorithms evaluation}

Aiming to quantify the performance of the heuristic algorithms in terms of stability and convergence guarantee, it is necessary to know the specified limits for the tolerances calculation, the so-called upper specification limit (USL) and lower specification limit (LSL) $[45,46]$.

The USL and LSL are simply an upper and lower bounds to measure the algorithm's performance. Thus, as in the case of control charts, it is desired that the algorithm behaves within these two limits. These parameters are often set by the need for quality of solutions 
found by the heuristic algorithm and may take values milder or stricter ${ }^{6}$. But this quality analysis should consider the acceptance limits of the solution, i.e., what are the thresholds of deviation from the desired value that can still be accepted as a solution for the optimization problem.

\subsection{Algorithm stability and capacity indexes}

One of the metrics widely used to evaluate the stability and capacity of the search algorithms is the so-called algorithm stability index (ASI), which corresponds to an ability measure of the algorithm to produce consistent results, described by the ratio between the dispersion of allowed solutions and the dispersion of current solution. The other metric, namely algorithm capability index (ACI) is a measure of how far from the specified limits the solution propitiates by the algorithm is, in terms of quality of the solutions obtained. The ASI and ACI can be calculated as:

$$
\begin{gathered}
A S I=\frac{(U S L-L S L)}{6 \sigma_{\bar{X}}} \\
A C I=\frac{(U S L-\bar{X})}{3 \sigma_{\bar{X}}} \quad \text { or } \quad A C I=\frac{(\bar{X}-L S L)}{3 \sigma_{\bar{X}}}
\end{gathered}
$$

In the literature, this methodology is also known as "Six Sigma" methodology [47]. The ACI metric measures how close the algorithm's solution is from its purpose, as well as the consistency around their average performance. An algorithm may have a minimal variation, but if it is away from the objective value for one of the specification limits, it will result in a lower ACI value, whereas the ASI metric may still be high. On the other hand, an algorithm could result, on average, in solutions exactly equal to the purpose, but presents a large variation in performance. In this case, ACI is still small and ASI can still be large. Thus, the ACI metric just will be large if and only if it reaches the vicinity of the desirable objective value consistently and with minimal variation.

Note that for practical reasons, it has been considered a good criterion to ensure $A S I>2$ and $A C I>1.33$ for most of engineering applications with practical interest $[46,48]$.

In the next section, the stability and capacity indexes have been considered in the input parameters optimization step of the heuristic algorithms, since the adopted benchmark functions have well-defined values for the global minimum, as described in the following.

\subsection{Input parameter optimization}

Considering the quality metrics discussed previously, it was decided to hold a Decathlon marathon type $[43,49]$ in order to evaluate the efficiency, stability an convergence capacity of the proposed heuristic $\mathrm{MuD}$ algorithms. For this purpose, ten benchmark functions, which correspond to the ten races of marathon, have been deployed aiming to define performance thresholds, as well as parameters determination that provide good solutions for all heuristic algorithms considered.

\footnotetext{
${ }^{6}$ A usual way is to take $U S L=\bar{X}+3 \sigma_{X}$ and $L S L=\bar{X}-3 \sigma_{X}$, where $\sigma_{X}$ is the standard deviation of the process $X$.
} 
Hence, in order to optimize the input parameters of each heuristic algorithm, ten benchmarks (test) functions described in Table 1 have been deployed, considering functions commonly used in the literature [34, 44,50-52], but with different characteristics in terms of local optima and dimensionality. In this study the first three functions of De Jong's work [50] have been considered, and in order to guarantee diversity in the characteristics, a set of seven additional test functions have been chosen.

\begin{tabular}{cc}
\hline Name & Definition \\
\hline De Jong [50] & $F 1(\mathbf{x})=\sum_{i=1}^{n} i \cdot x_{i}^{2}$ \\
\hline De Jong [50] & $F 2(\mathbf{x})=\sum_{i=1}^{n-1} 100\left(x_{i-1}-x_{i}^{2}\right)^{2}+\left(1-x_{i}\right)^{2}$ \\
\hline De Jong [50] & $F 3(\mathbf{x})=\sum_{i=1}^{n}\left\lfloor x_{i}\right\rfloor$ \\
\hline Michalewicz [52] & $F 4(\mathbf{x})=-\sum_{i=1}^{n} \sin \left(x_{i}\right)\left(\sin \left(\frac{i x_{i}^{2}}{\pi}\right)\right)^{2 m}$ \\
\hline Schaffer [53] & $F 5(\mathbf{x})=\left(x_{1}^{2}+x_{2}^{2}\right)^{0,25}\left(\sin ^{2}\left(50\left(x_{1}^{2}+x_{2}^{2}\right)^{0,1}\right)+1\right)$ \\
\hline Ackley [54] & $F 6(\mathbf{x})=x_{1}^{2}+2 x_{2}^{2}-0,3 \cos \left(3 \pi x_{1}\right)-0,4 \cos \left(4 \pi x_{2}\right)+0,7$ \\
\hline Rastrigin [55] & $F 7(\mathbf{x})=A n+\sum_{i=1}^{n}\left[x_{i}^{2}-A \cos \left(2 \pi x_{i}\right)\right]$ \\
\hline Schwefel [56] & $F 8(\mathbf{x})=A n+\sum_{i=1}^{n}\left[-x_{i} \sin \left(\sqrt{\left|x_{i}\right|}\right)\right]$ \\
\hline 6-Hump Camelback [57] & $F 9(\mathbf{x})=\left(4-2,1 x_{1}^{2}+\frac{x_{1}^{4}}{3}\right) x_{1}^{2}+x_{1} x_{2}+\left(-4+4 x_{2}^{2}\right) x_{2}^{2}$ \\
\hline Shubert [58] & $F 10(\mathbf{x})=\prod_{i=1}^{n}\left(\sum_{j=1}^{m} j \cos \left[(j+1) x_{i}+j\right]\right)$ \\
\hline
\end{tabular}

Table 1. Benchmark functions deployed for the heuristic input parameters optimization.

In order to eliminate eventual bias in the analysis, a large number of simulations have been considered. Hence, in all numerical results presented in this work an average over at least 1000 realization for each numerical parameter determination of each algorithm and for each function have been carried out, aiming to determine means and respective standard deviations, as well as for the calculation of the ASI and ACI quality measures. Thus, the numerical results show confidence intervals that provide consistent analyzes.

As a result of these analyzes, Table 2 presents a summary for the input parameters optimization and adopted strategies (in order to guarantee diversity on the search space) for each (hyper)-heuristic algorithm analyzed in this work. $Q_{\text {indiv }}$ indicates the length of each individual-candidate solution which of course is a function of the problem dimensionality.

\subsection{Computational complexity}

Table 3 presents the generic complexity of the heuristic algorithms for subsequent determination of the heuristic multiuser detectors (MuD) complexity operating under different telecommunications systems scenarios in addition to the presentation of quantitative computational complexity of algorithms for application in Decathlon proof considered in this work (F1 to F10 functions). Notation: $\mathcal{O}_{F C}$ represents the number 


\begin{tabular}{lll}
\hline & Population Size: & $p=10 \cdot[0.345(\sqrt{\pi(l-1)}+2)]$ \\
& Mating Pool Size: & $T=0.7 p$ \\
GA & Selection Strategy: & $p$-sort \\
& Crossover Type/Rate: & Uniform $/ p_{c}=50 \%$ \\
& Mutation Type/Rate: & Gaussian $/ p_{m}=10 \%$ \\
& Replacement Strategy: & $\mu+\lambda($ with $\mu=p)$ \\
\hline \multirow{4}{*}{ EP-C } & Population Size: & $p=10 \cdot[0.345(\sqrt{\pi(l-1)}+2)]$ \\
& Cloning Rate: & $I_{\mathcal{C}}=20 \%$ \\
& Selection Strategy: & $p$-sort \\
& Mutation Type/Rate: & Gaussian $/ p_{m}=15 \%$ \\
& Replacement Strategy: & $\mu+\lambda($ with $\mu=p)$ \\
\hline \multirow{k}{*}{-opt } & Neighborhood Search & Choose neighborhood size $(k)$ \\
\multirow{3}{*}{ SA } & Initial temperature: & $T(0)=\ln \left(I_{t}\right)$ \\
& Step Size (Plateau): & $L_{s a}=2$ \\
& Cooling Step: & $\varepsilon=\sqrt{\frac{2}{\ln (i)}}$ \\
& Acceptance Probability: & $x(i)=\exp \left[\frac{|\Delta e|}{T(i)}\right]-1$ \\
\hline STTS & Prohibition Period: & $P=Q_{\text {indiv }} / 2$ \\
\hline \multirow{2}{*}{ RTS } & Initial Prohibition Period: & $P(0)=Q_{\text {indiv }} / 2$ \\
& Reduction/Increase Rate: & $x=50 \%$ \\
\hline \multirow{2}{*}{ HH } & Number of HH iteration: & $I_{t}(H H)=10$ \\
& Selection Strategy: & Simply random \\
& Acceptance Strategy: & Naive \\
\hline
\end{tabular}

Table 2. A summary for the optimized input parameters and strategies adopted in all considered heuristic algorithms

of operations relevant to the cost function calculation, and $G_{t}$ is the number of iteration/generation necessary for convergence.

\begin{tabular}{lcc}
\hline & Flops & Foremost Term \\
\hline GA & $p G_{t}\left(\mathcal{O}_{F C}+11,7 Q_{\text {indiv }}+3 \log \left(Q_{\text {indiv }}\right)\right)$ & $p G_{t} \mathcal{O}_{F C}$ \\
\hline EP-C & $p G_{t}\left(\mathcal{O}_{F C}+6,1 Q_{\text {indiv }}+3 \log \left(Q_{\text {indiv }}\right)\right)$ & $p G_{t} \mathcal{O}_{F C}$ \\
\hline 1-opt & $Q_{\text {indiv }} G_{t}\left(\mathcal{O}_{F C}+2 Q_{\text {indiv }}+2\right)$ & $Q_{i n d i v} G_{t} \mathcal{O}_{F C}$ \\
\hline$k$-opt & $\sum_{i=1}^{k}\left(\begin{array}{c}Q_{\text {indiv }} \\
i\end{array}\right) G_{t}\left(\mathcal{O}_{F C}+2 Q_{\text {indiv }}+2\right)$ & $\sum_{i=1}^{k}\left(\begin{array}{c}Q_{\text {indiv }} \\
i\end{array}\right) G_{t} \mathcal{O}_{F C}$ \\
\hline SA & $Q_{\text {indiv }} G_{t}\left(\mathcal{O}_{F C}+3 Q_{\text {indiv }}+5\right)$ & $Q_{\text {indiv }} G_{t} \mathcal{O}_{F C}$ \\
\hline STTS & $Q_{\text {indiv }} G_{t}\left(\mathcal{O}_{F C}+3,5 Q_{\text {indiv }}+\log \left(Q_{\text {indiv }}\right)+6\right)$ & $Q_{\text {indiv }} G_{t} \mathcal{O}_{F C}$ \\
\hline RTS & $Q_{\text {indiv }} G_{t}\left(\mathcal{O}_{F C}+3,5 Q_{\text {indiv }}+\log \left(Q_{\text {indiv }}\right)+7\right)$ & $Q_{\text {indiv }} G_{t} \mathcal{O}_{F C}$ \\
\hline HH & $\left(0,6 Q_{\text {indiv }}+0,4 p\right) G_{t} \mathcal{O}_{F C}+$ \\
\multicolumn{3}{c}{$\left(0,6 Q_{\text {indiv }}+0,4 p\right) G_{t} \mathcal{O}_{F C}$} \\
\hline
\end{tabular}

Table 3. Average complexity in terms of number of operations (Flops) and predominant term, for all considered heuristic algorithms. 


\section{Numerical results for DS/CDMA systems with multidimensional diversity}

This section discuss representative numerical results for multiuser detection obtained under various types of diversity scenarios. First, we present results for a SIMO MC-CDMA systems, i.e. systems in which frequency and space diversities have been deployed jointly, due to the use of multicarrier and multiple receiving antennas. In a second step, a scenario with code and spatial diversity using multiple receive and/or transmission antennas have been analyzed.

The main parameters of the system and channel coefficients are presented in Table 4. In all simulations results, random spreading sequences and slow Rayleigh channel model have been considered. Furthermore, it was assumed that the channel parameters are perfectly known at the receiver side, as well each subcarrier of the MC-CDMA system is subjected to flat frequency fading. Besides, low (BPSK) and high order modulation (M-QAM) format, LDPC and Turbo coding, as well as different spreading codes length, ranging from $N=8$ to $N=64$ have been deployed in this section.

\begin{tabular}{lccccc}
\hline Parameters & Fig.2 & Fig.3 & Fig.4 & Fig.5 & Fig. 6 \\
\hline \# Users & 20 & 20 & 1 and 32 & 1 and 32 & 64 \\
\hline \# Antenas Tx & 1 & 1 & 4 & 4 & 1 and 2 \\
\hline \# Antenas Rx & 1 to 4 & 1 to 5 & 1 & 4 & 1 and 2 \\
\hline Modulation & BPSK & BPSK & M-QAM & M-QAM & BPSK \\
\hline Spread Sequence & $N=8$ & $N=8$ & $N=32$ & $N=32$ & $N=64$ \\
\hline Subcarriers & $M=4$ & $M=4$ & - & - & $M=64$ \\
\hline SNR $(\gamma)$ & $9 \mathrm{~dB}$ & 0 to $18 \mathrm{~dB}$ & -2 to 32dB & -10 to $24 \mathrm{~dB}$ & 0.5 to 5dB \\
\hline Max. Doppler Freq. & $100 \mathrm{~Hz}$ & $100 \mathrm{~Hz}$ & $20 \mathrm{~Hz}$ & $20 \mathrm{~Hz}$ & $30 \mathrm{~Hz}$ \\
\hline Channel & - & - & short LDPC & short LDPC & Turbo \\
Coding & - & - & $(204,102)[59]$ & $(204,102)[59]$ & $(R=1 / 2)$ \\
\hline Channel & - & - & Belief & Belief & Turbo \\
Decoding & - & - & Propag. & Propag. & (MAP) \\
\hline Space-Time & - & - & Rate 1 & Rate 1 & Rate 1 \\
Coding & - & - & $R_{S T B C}=1[4]$ & $R_{S T B C}=1[4]$ & $R_{S T B C}=1[60]$ \\
\hline
\end{tabular}

Table 4. Adopted channel and multicarrier multiple-antenna system parameters.

Figures 2 and 3 consider systems with space and frequency diversity, in SIMO MC-CDMA scenarios. Monte Carlo simulation results indicate that the GA, SA, RTS, STTS, EP-C and HH multiuser detectors result in the same near-optimal performance in terms of solution quality after convergence, but with different complexities. However, the local search algorithm (1-LS and 3-LS) presented ACI and ASI measures below desirable thresholds and are not suitable for applications in scenarios of multiuser detection with multidimensional diversity.

Figure 2 shows the convergence behavior as a function of the number of iterations for all heuristic $\mathrm{MuD}$ algorithms considered. Note the equality of BER performance achieved after total convergence for all heuristic techniques. Specifically for convergence evaluation, different initial solutions were considered, while all achieving performances significantly superior to the Conventional detector. However, the number of generations/iterations for convergence proved to be different, which will be analyzed in details on Section 5.1 and 5.2. Note that increasing the number of receiving antennas implies in a significant 
performance improvement (due to the spatial diversity) for a medium loading system and low signal-to-noise ratio (SNR).

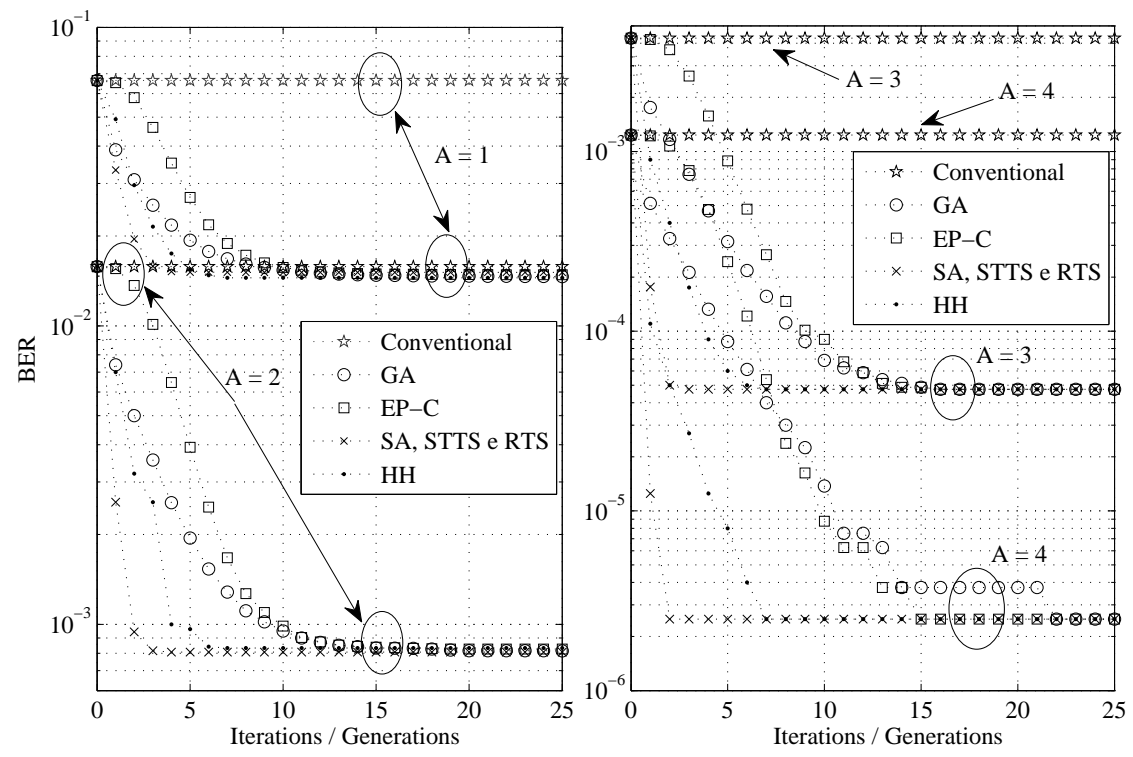

Figure 2. Convergence speed for the conventional and various heuristic algorithm detectors under SIMO MC-CDMA Scenarios with $E_{b} / N_{0}=9 \mathrm{~dB}$ and $K=20$ users.

Figure 3 shows the BER performance for SIMO MC-CDMA with different number of receiving antennas $\left(A_{R x}=1,2, \ldots 5\right)$ and medium loading system $(K=20)$. Accordingly, a $B E R=10^{-5}$ for a moderate number of $A_{R x}$ antennas and SNR has been achieved. Thus, there is an expressive performance gain with heuristic $\mathrm{MuD}$ strategies regarding the Conventional detector when the number of antennas is increased for signal-to-noise ratio in the range $[0 ; 18] \mathrm{dB}$.

Systems with multiple-input-multiple-output (MIMO) and space-time block code (STBC) represent a promising solution often incorporated in commercial standards such as Wimax. Furthermore, a better performance $\times$ complexity trade-off can be obtained through the use of low density parity check codes (LDPC). The choice of STBC topology should take into account performance criteria, such as coding gain, diversity gain, multiplexing gain, and obviously the decoder complexity. However, these topics are not the focus of the this work and, therefore, more information can be found in the references $[4,60]$.

The considered MIMO system is formed by $A_{T x}=4$ transmit antennas and $A_{R x} \geq 1$ receiving antennas, with 4 symbols transmitted simultaneously. Furthermore, the following parameters have been adopted (see Table 4): $A_{T x} A_{R x}$ flat fading statistically independent channels, $M-$ QAM modulation, quasi-orthogonal STBC (QO-STBC) scheme with rate 1 [4], short $\operatorname{LDPC}(204,102)$ code, perfect channel state information knowledge at receiver, random sequences of length $N=32$ and two scenarios with loading system $\mathcal{L}=\frac{K}{N}=\frac{1}{32}$ and 1 . 


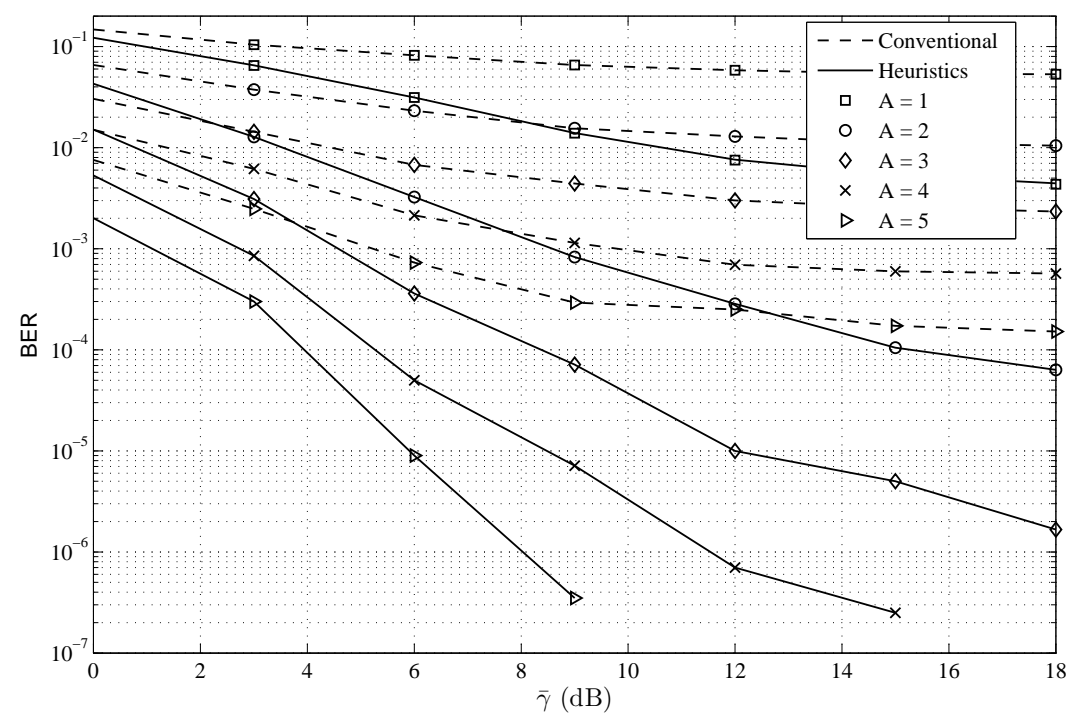

Figure 3. BER performance for heuristic algorithms. SIMO MC-CDMA system with $K=20$ users.

Figure 4 depicts the BER performance versus SNR at the receiver input for different modulation constellations with $A_{T x}=4$ and $A_{R x}=1$ antennas. As expected, the single-user performance achieves very low $\mathrm{BER}^{7}$ under smaller SNR than that necessary with high loading system (with $K=32$ users). However, it is observed that with an increment of 2-3dB in SNR for systems with high loading it is possible to obtain very lower BER, especially for 4-QAM modulation. However, higher order modulations such as 256-QAM enable the transmission of more bits per symbol period, which result in higher throughput systems if more power/energy is available at transmitter. Furthermore, the performance loss by increasing the loading proves be small, enabling the deployment of heuristic MuD algorithms in coded CDMA systems.

Figure 5 compares the heuristic MuD performance in terms of BER versus SNR for high order modulation constellations and considering $A_{T x}=4$ and $A_{R x}=4$ antennas. We observe the same behavior shown in Figure 4. But in this case the number of receiving antennas has been increased to $A_{R x}=4$, resulting in significant performance improvement with reduction of $\approx 10 \mathrm{~dB}$ SNR requirement, in order to obtain similar BERs. Again, the performance loss under total loading system is marginal; as a result, all heuristic algorithms discussed herein can be considered suitable for multiuser detection in coded CDMA systems. It is noteworthy that all heuristics algorithms showed the same level of BER performance after 40 generations for GA and EP-C, and 45 iterations for the SA, STTS, RTS and HH algorithms.

Figure 6 shows the BER performance of a multicarrier DS/CDMA (MC-CDMA) system with various types of diversity, considering multiple-input-multiple-output (MIMO) STBC coding, encoding and decoding turbo. This topology ${ }^{8}$ was adopted in order to represent a transmission-reception topology with a great diversity order, making possible to obtain

\footnotetext{
7 Beyond a certain SNR value, the performance improves sharply.

${ }^{8}$ Several other topologies can be considered for multidimensional analysis. For details, please see [61].
} 


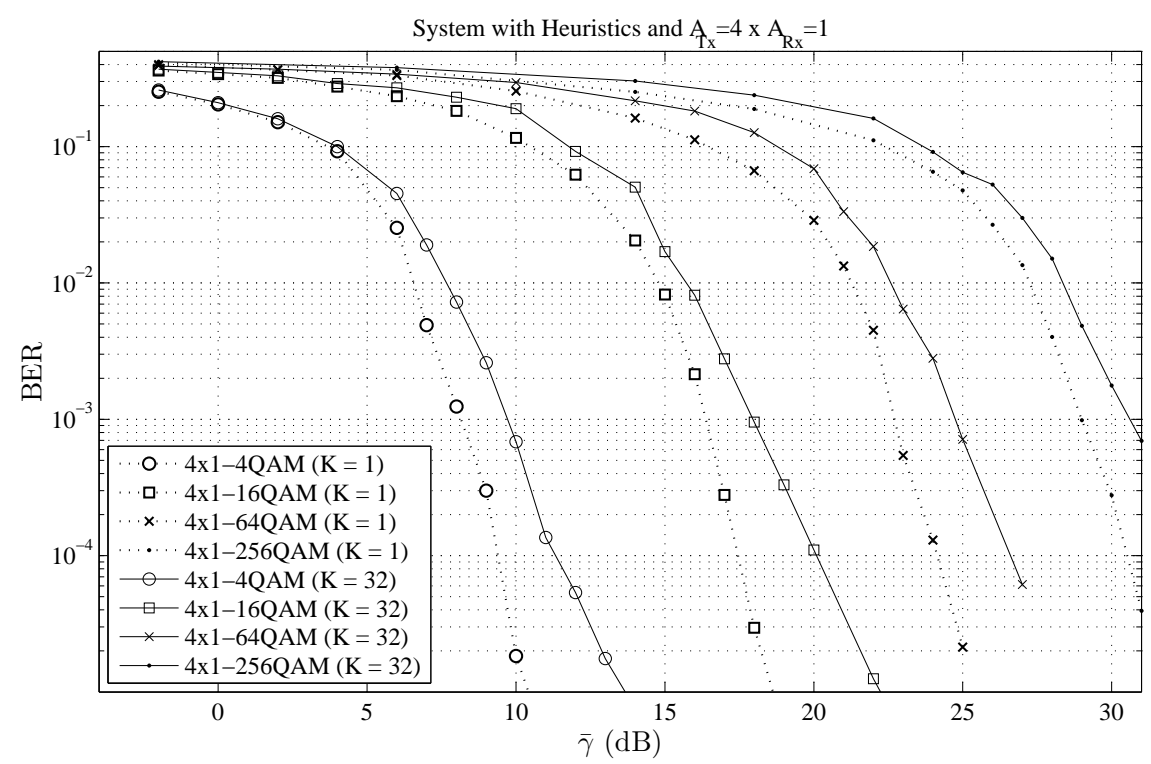

Figure 4. BER performance of the heuristic decoders for QO-STBC MIMO systems with short LDPC(204,102), $A_{T x}=4, A_{R x}=1$ antennas and $K=1$ or $K=32$ users.

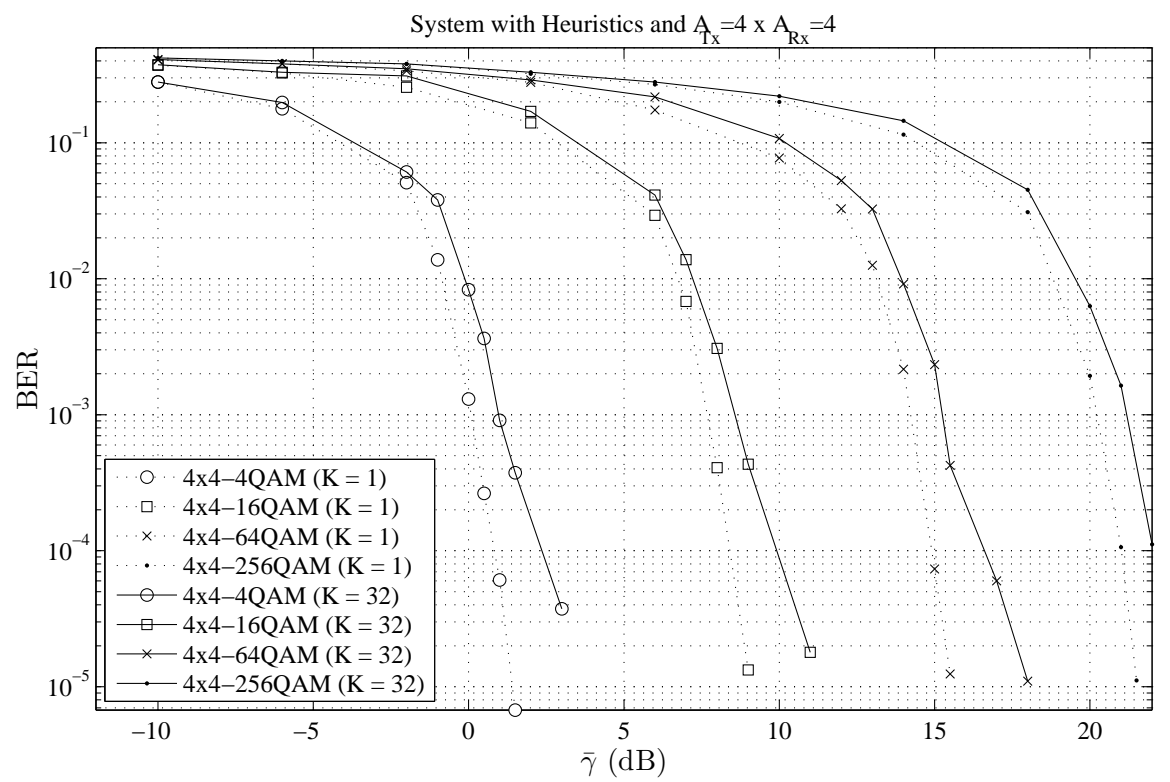

Figure 5. BER performance of the heuristic decoders for QO-STBC MIMO systems with short coding $\operatorname{LDPC}(204,102), A_{T x}=4$, $A_{R x}=4$ antennas and $K=1$ or $K=32$ users. 
excellent BER performance even for low SNR region. Of course, this topology is promising for adoption as a commercial standard.

Specifically, in the performance of Figure 6, a Turbo encoding and decoding of rate 1/2, which result in a spectral efficiency of $0.5 \mathrm{bps} / \mathrm{Hz}$ have been adopted. Again, there is a remarkable performance gain increasing when the transmit-receive antenna array becomes larger for both MMSE and heuristic multiuser detectors. Another important aspect to be mentioned is the extremely low BERs for $\bar{\gamma} \leq 3 \mathrm{~dB}$ achieved with heuristics MuD topologies even under $1 \times 1$ antenna array configuration. Increasing the antenna array to $2 \times 2$ and adopting Alamouti code [60] it was possible to obtain a performance of $B E R \leq 10^{-4}$ even for $\bar{\gamma} \leq 2 \mathrm{~dB}$. Thus, the performance achieved by MuD heuristics topology approaches the single-user bound (SuB) demonstrating the huge potential applicability in commercial communication systems and standards, specially those ones with high-performance and reliability requirements.

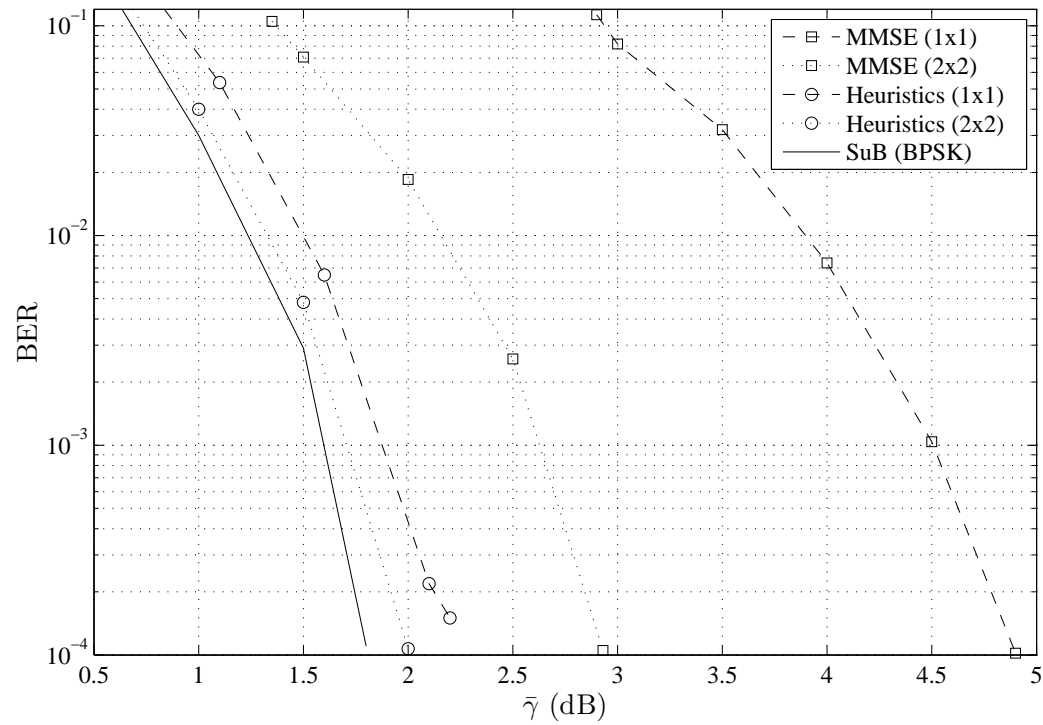

Figure 6. BER performance against SNR for a system with turbo channel coding.

Moreover, different topologies of the chosen one can be analyzed by considering, for example, channel coding (Convolutional or LDPC codes) and spatial diversity with other settings. However, the purpose of this section is to validate the potential of application of heuristics in $\mathrm{MuD}$ scenarios with multidimensional diversity and not compare topologies and system settings.

It is noteworthy that all heuristic $\mathrm{MuD}$ algorithms showed the same BER performance level after 100 generations for GA and EP-C algorithms, and 120 iterations for the SA, STTS, RTS and $\mathrm{HH}$ algorithms. 
The presented results considering multiuser detection with different level of diversity exploitation in (non)coded telecommunication systems have demonstrated the effective applicability of the proposed heuristics MuD techniques, due to significant improvement in BER with reduced complexity regarding the optimal multiuser detector $(\mathrm{OMuD})$. Complexity aspects of the proposed heuristic MuDs are discussed in the next section.

\subsection{Overall systems complexity}

As the complexity of the algorithms in terms of number of operations has been determined, one can determine the complexity of each telecommunication scenario considering the complexity for the cost function calculation.

Both terms of the cost function, defined by $\mathcal{F}_{1}=\mathcal{C}^{H} \mathcal{A Y}$ and $\mathcal{F}_{2}=\mathcal{C} \mathcal{A} \mathbf{R} \mathcal{A C}{ }^{H}$ in eq. (14) can be obtained before the loop optimization in each MuD heuristic algorithm. Thus, for each candidate-solution evaluation, $\mathcal{B}^{T} \mathcal{F}_{1}$ and $\mathcal{B}^{T} \mathcal{F}_{2} \mathcal{B}$ are computed, which in terms of operations is equivalent to $(K I D)^{2}+4 K I D$ operations. For $\mathrm{OMuD}$ detector, the number of operations grows exponentially with the number of users, i.e., $\mathcal{O}\left(2^{K I}(K I D)^{2}\right)$. It takes $2^{K I}$ bit generations of order $K I D$, as well as $2^{K I}$ cost function calculations for the simultaneous detection of a frame consisting of $I$ bits of $K$ users on a system with multiuser detection operating on fading channels.

Therefore, in order to calculate the cost function, $\mathcal{O}_{F C}=(K I D)^{2}+4 K I D$ operations are needed. It is noteworthy that 42 different scenarios have been analyzed ${ }^{9}$. Besides, scores to define the best strategy have been considered this metric in different scenarios.

Thus, the computational complexity of each proposed heuristic MuD algorithm, in terms of number of operations, has been obtained under different operation scenarios. Table 5 presents such complexities. Strategy with the lowest complexity using the adopted scoring system, for each analyzed scenario has been indicated with bold numbers. It is noteworthy that the scores were normalized considering the higher value with score of 100. For sake of comparison, the optimal MuD complexity is presented in the last column.

\begin{tabular}{|c|c|c|c|c|c|c|c|c|}
\hline Scenario & & $\overline{\text { GA }}$ & EP-C & SA & STTS & RTS & HH & OMuD \\
\hline 1 - Fig. 2 & $\left(\times 10^{6}\right)$ & 4,597 & 4,328 & 5,572 & 5,606 & 5,607 & 5,144 & $8,22.10^{21}$ \\
\hline 2 - Fig. 3 & $\left(\times 10^{8}\right)$ & 2,322 & 2,303 & 3,333 & 3,336 & 3,336 & 2,926 & $1,52.10^{45}$ \\
\hline 3 - Fig. 4 & $\left(\times 10^{9}\right)$ & 4,390 & 4,382 & 5,794 & 5,795 & 5,795 & 5,451 & $1,03.10^{57}$ \\
\hline 4 - Fig.5 & $\left(\times 10^{13}\right)$ & 2,569 & 2,569 & 18,877 & 18,878 & 18,878 & 12,483 & $>10^{300}$ \\
\hline 5 - Fig. 6 & $\left(\times 10^{13}\right)$ & 1,028 & 1,027 & 22,277 & 22,278 & 22,278 & 13,860 & $>10^{300}$ \\
\hline Score & & 87 & 100 & 83 & 72 & 56 & 85 & - \\
\hline Position & & $2^{\text {nd }}$ & $1^{\text {st }}$ & $4^{\text {th }}$ & $5^{\text {th }}$ & $6^{\text {th }}$ & $3^{\text {rd }}$ & - \\
\hline
\end{tabular}

Table 5. Necessary number of operations in all optimized Scenarios.

\subsubsection{Computational Time}

Furthermore, the complexity in terms of computational time has been determined for each telecommunication scenario. As a result, the computational time for calculating one cost function according each specific scenario has been quantified. We have deployed a personal

\footnotetext{
${ }^{9}$ But not shown herein due to the lack of space. Additional results can be checked in [61].
} 
computer with the following configuration: Motherboard ASUS P8H67-M EVO, Intel I7-2600 with 3.4 GHz clock and 8MB cache; Memory 8GB Corsair Dominator DDR3 1333MHz and a Video board Radeon HD6950 2GB DDR5.

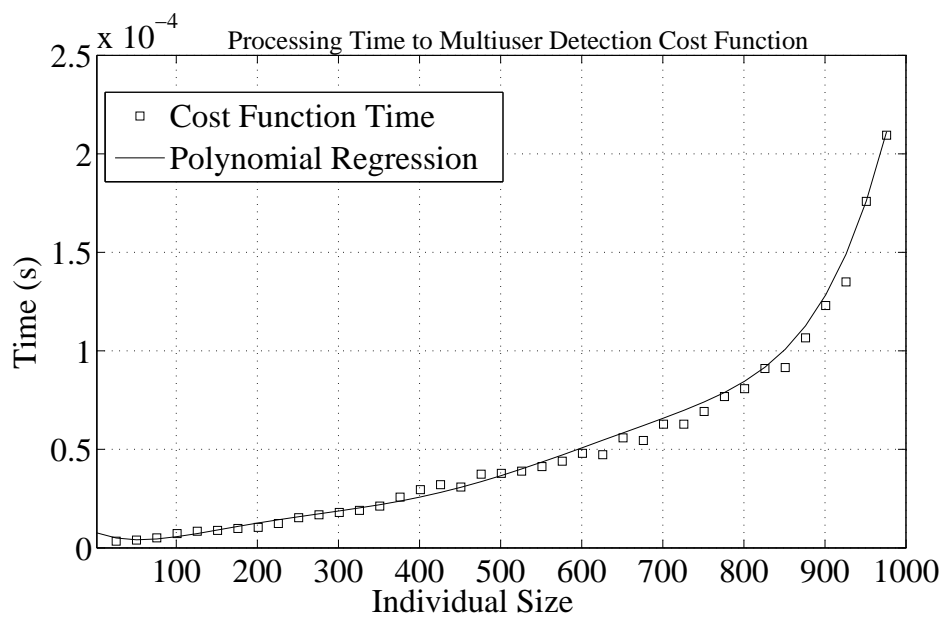

Figure 7. Average time for cost function calculation and respective polynomial approximation.

Figure 7 depicts the time to calculate a cost function as the size of the individual increases, while Table 6 shows the average time required for optimization algorithms in each scenario considered, as well as the respective scores and classification.

\begin{tabular}{|c|c|c|c|c|c|c|}
\hline Scenario & $\overline{\text { GA }}$ & EP-C & $\overline{\mathrm{SA}}$ & STTS & RTS & $\mathrm{HH}$ \\
\hline 1 - Fig.2 & 0,039 & 0,0378 & 0,0474 & 0,0477 & 0,0477 & 0,0436 \\
\hline 2 - Fig.3 & 0,297 & 0,2892 & 0,7509 & 0,7532 & 0,7538 & 0,5948 \\
\hline 3 - Fig. 4 & 0,4638 & 0,4515 & 1,1704 & 1,1741 & 1,1749 & 0,9414 \\
\hline 4 - Fig.5 & 128,126 & 128,001 & 914,891 & 914,876 & 914,906 & 609,049 \\
\hline 5 - Fig. 6 & 5,2576 & 5,1283 & 24,0933 & 24,1408 & 24,1608 & 18,2711 \\
\hline Score & 88 & 100 & 73 & 66 & 56 & 80 \\
\hline Position & $2^{\text {nd }}$ & $1^{\text {st }}$ & $4^{\text {th }}$ & $5^{\text {th }}$ & $6^{\text {th }}$ & $3^{\text {rd }}$ \\
\hline
\end{tabular}

Table 6. Average time (in seconds) spend by the optimized heuristic algorithms under Scenarios $1-5$.

In conclusion, again the algorithm EP-C presents the lowest complexity in terms of computational time. Note that all heuristic algorithms result in a computational time, as well as number of operation very close each other. Thus, we can adopt any topologies without significant loss in terms of performance $\times$ complexity trade-off metric, validating the deployment of heuristic MuD approach with optimized input parameters as shown in Table 2.

\subsection{Quality, stability and topology choice}

For all scenarios considered, convergence curves in terms of BER have been resulted in the same level achieved for all algorithms, but after a different number of generations or 
iterations ${ }^{10}$. In order to evaluate the stability of the algorithms after convergence, some tests have been conducted for a large number of generations/iterations. For all algorithms, the average performance level and number of generations/iterations for total convergence hold under the same boundaries.

Therefore, for the multiuser detection problems presented in this work it is concluded that the proposed heuristic algorithms are able to find the optimum solution or very near-optimum solution with a reduced number of cost function tests, approaching the MuD performance since an adequate and sufficient number of generations/iterations has been available. In this context we are interest in analyze the variation of the solutions when decreasing the number of generations/iterations; as a result, the heuristic MuD algorithms were also classified following the criterion of the smaller deviation values of solutions.

In addition, for the complexity analysis, we chose to average between scores (number of operations and computational time), thus avoiding an unfair analysis among optimization strategies or possible bias.

Table 7 presents the classification for the six heuristic MuD with the adoption of equal weight features for the final score calculation. In conclusion, under equal weight metric for computational complexity and quality/stability of the solutions found, the following choice criteria for MuD problem can be established:

\begin{tabular}{lc}
\hline Criterion & Algorithm Choice \\
\hline Best BER performance: & HH \\
Lowest Complexity: & EP-C \\
Performance-Complexity trade-off: & HH (score: 91) \\
\hline
\end{tabular}

\begin{tabular}{ccccccc}
\hline Score & GA & EP-C & SA & STTS & RTS & HH \\
\hline Number of Operation & 87 & 100 & 83 & 72 & 56 & 85 \\
Computational Time & 88 & 100 & 73 & 66 & 56 & 80 \\
\hline Complexity (Average) & 88 & 100 & 78 & 69 & 56 & 83 \\
\hline & & & & & & \\
\hline Quality and Stability & 80 & 67 & 78 & 64 & 62 & 100 \\
\hline Final Score (Average) & 84 & 83 & 78 & 66 & 59 & 91 \\
Final Position & $2^{\text {nd }}$ & $3^{\text {rd }}$ & $4^{\text {th }}$ & $5^{\text {th }}$ & $6^{\text {th }}$ & $1^{\text {st }}$ \\
\hline
\end{tabular}

Table 7. Scores for all considered heuristic algorithms applicable to wireless communication Scenarios $1-5$.

\section{Main conclusions}

Several heuristic techniques applied to multiuser detection problems under different channel and system configuration scenarios, as well as diversity dimensionality, such as time, frequency, space and coding have been analyzed in this work. The main purpose in

\footnotetext{
${ }^{10}$ This result was obtained when the initial inputs guess for all algorithms considered are the same, indicating that all algorithms were able to achieve convergence after a certain number of generations/iterations, except the LS algorithm, which did not show enough stability for adoption.
} 
combining different types of diversity with heuristic detection is to provide system capacity increasing and/or reliability improvement.

Near single-user bound performance has been achieved by all MuD heuristic algorithms analyzed in this work, considering different system and channel configurations, while offer a dramatic complexity reduction regarding the $\mathrm{OMuD}$ with marginal performance loss, even in aggressive fading channels and high loading systems conditions.

Among the analyzed detectors, the best MuD heuristic algorithm choice must take into account that one which offer either smallest computational complexity or the best BER performance, i.e. EP-C or HH multiuser detectors, respectively. Hence, the criteria for topology ranking established in this work allow us to quantify the parameter optimization level, reflecting on the quality and stability of the solutions obtained.

The heuristic input parameter optimization, as well as the proposed methodology for the heuristic $\mathrm{MuD}$ topology choice represent the main contribution of this work. Under optimized input parameters condition of all heuristic MuD algorithms, the quality and stability analyses have been carried out deploying ten benchmark functions. The numerical results for the $\mathrm{MuD}$ problem confirmed the near-optimal performance achieved by the heuristic algorithms for a wide channel and system configurations, corroborating the methodology adopted for the ranking topology.

\section{Acknowledgements}

This work was supported in part by the National Council for Scientific and Technological Development (CNPq) of Brazil under Grants 202340/2011-2, 303426/2009-8 and in part by Londrina State University - Paraná State Government (UEL).

\section{Author details}

Fernando Ciriaco $^{1, \star}$,

Taufik Abrão ${ }^{1}$ and Paul Jean E. Jeszensky ${ }^{2}$

^ Address all correspondence to: fciriaco@uel.br; abrao@ieee.org; pjj@lcs.poli.usp.br

1 Electrical Engineering Department, State University of Londrina (DEEL-UEL), Londrina, Paraná, Brazil

2 Polytechnic School of the University of Sao Paulo (EPUSP), Sao Paulo, Brazil

\section{References}

[1] R. L. Peterson, R. E. Ziemer, and D. E. Borth. Introduction to Spread Spectrum Communications. Prentice Hall, New Jersey, 1 edition, 1995.

[2] C. E. Shannon. A mathematical theory of communication. Bell System Technical Journal, 27:379-423 and 623-656, July and October 1948.

[3] S. Verdú. Multiuser Detection. Cambridge University Press, USA, 1998.

[4] H. Jafarkhani. Space-Time Coding: Theory and Practice. Cambridge University Press, 2005. 
[5] D. Tse and P. Viswanath. Fundamentals of Wireless Communication. Cambridge University Press, Cambridge, UK, 2005.

[6] S. Verdú. Minimum probability of error for asynchronous gaussian multiple-access channels. IEEE Transactions on Information Theory, 32:85-96, January 1986.

[7] A. Duel-Hallen, J. Holtzman, and Z. Zvonar. Multiuser detection for cdma systems. IEEE Personal Communications, pages 46-58, April 1995.

[8] H. V. Poor and S. Verdú. Probability of error in mmse multi-user detection. IEEE Transactions on Information Theory, 43(3):858-881, May 1997.

[9] P. Patel and J. M. Holtzman. Analysis of a single sucessive interference cancellation scheme in a ds/cdma system. IEEE Journal on Selected Areas in Communications, 12:796-807, June 1994.

[10] R. Agarwal, B. V. R. Reddy, and K. K. Aggarwal. A reduced complexity hybrid switched mode detector using interference cancellation methods for ds-cdma systems. In Annual IEEE India Conference, pages 1 - 4, September 2006.

[11] A. Duel-Hallen. Decorrelating decision-feedback multiuser detector for synchronous cdma channel. IEEE Transactions on Communications, 41(2):285-290, February 1993.

[12] P. H. Tan. Multiuser detection in cdma-combinatorial optimization methods. Master's thesis, Chalmers University of Technology, Göteborg, 2001.

[13] F. Ciriaco, T. Abrão, and P. J. E. Jeszensky. Ds/cdma multiuser detection with evolutionary algorithms. Journal of Universal Computer Science, 12(4):450-480, 2006.

[14] S. Abedi and R. Tafazolli. Genetically modified multiuser detection for code division multiple access systems. IEEE Journal on Selected Areas in Communications, 20(2):463-473, February 2001.

[15] T. Abrão, F. Ciriaco, and P. J. E. Jeszensky. Evolutionary programming with cloning and adaptive cost funciton applied to multi-user ds-cdma systems. In 2004 IEEE International Symposium on Spread Spectrum Techniques and Applications, pages 160-163, Sydney, August 2004. Australia.

[16] K. Yen and L. Hanzo. Genetic-algorithm-assisted multiuser detection in asynchronous cdma communications. IEEE Transactions on Vehicular Technology, 53(5):1413-1422, September 2004.

[17] F. Ciriaco, T. Abrão, and P. J. E. Jeszensky. Multirate multiuser ds/cdma with genetic algorithm detection in multipath channels. In 2006 IEEE International Symposium on Spread Spectrum Techniques and Applications, Manaus, August 2006. Brazil.

[18] M. S. Arifianto, A. Chekima, L. Barukang, and M.Y. Hamid. Binary genetic algorithm assisted multiuser detector for stbc mc-cdma. In International Conference on Wireless and Optical Communications Networks, pages 1 - 5, July 2007. 
[19] R. Zhang and L. Hanzo. Harmony search aided iterative channel estimation, multiuser detection and channel decoding for ds-cdma. In Vehicular Technology Conference Fall, pages 1 - 5, September 2010.

[20] P. J. E. Jeszensky and G. Stolfi. Cdma systems sequences optimization by simulated annealing. In IEEE 5th International Symposium on Spread Spectrum Techniques and Applications, volume 3, pages 706-708, Sun City, 1998. South Africa.

[21] T. M. Chan, S. Kwong, K. F. Man, and K. S. Tang. Sequences optimization in ds/cdma systems using genetic algorithms. In IEEE Region 10 International Conference on Electrical and Electronic Technology, volume 2, pages 728-731, Phuket Island, August 2001. Singapore.

[22] F. Ciriaco, T. Abrão, and P. J. E. Jeszensky. Genetic algorithm applied to multipath multiuser channel estimation in ds/cdma systems. In 2006 IEEE International Symposium on Spread Spectrum Techniques and Applications, Manaus, August 2006. Brazil.

[23] T. Yano, K. Nakamura, T. Tanaka, and S. Honda. Channel parameter estimation in the cdma multiuser detection problem. In Fourth International Conference on Networked Sensing Systems, June 2007.

[24] D. Zhao, M. Elmusrati, and R. Jantti. On downlink throughput maximization in ds-cdma systems. In IEEE 61st Vehicular Technology Conference, volume 3, pages 1889 - 1893, May 2005.

[25] F. R. Durand, L. Melo, L. R. Garcia, A. J. dos Santos, and Taufik Abrão. Optical network optimization based on particle swarm intelligence. In Taufik Abrão, editor, Search Algorithms, volume 1, pages 1-21. InTech Open, 2012.

[26] M. Moustafa, I. Habib, and M. N. Naghshineh. Efficient radio resource control in wireless networks. IEEE Transactions on Wireless Communications, 3:2385-2395, November 2004.

[27] T. Abrão, L. D. H. Sampaio, M. L. Proença Jr, and P. J. E. Angélico, B. A.; Jeszensky. Multiple access network optimization aspects via swarm search algorithms. In Nashat Mansour, editor, Search Algorithms and Applications, volume 1, pages 261-298. InTech Open, 2011.

[28] M. P. Marques, M. H. Adaniya, T. Abrão, L. H. D. Sampaio, and Paul Jean E. Jeszensky. Ant colony optimization for resource allocation and anomaly detection in communication networks. In Taufik Abrão, editor, Search Algorithms, volume 1, pages 1-34. InTech Open, 2012.

[29] J. G. Proakis. Digital Communications. Electrical Engineering. Communications and Signal Processing. McGraw-Hill, New York, 2 edition, 1995.

[30] S. Verdú. Computational complexity of optimum multiuser detection. Algorithmica, 4:303-312, 1989. 
[31] C. R. Darwin. On The Origin of Species by Means of Natural Selection. John Murray, London, 1859.

[32] C. R. Darwin. Origin of Species. Wilder Publications, 2008.

[33] J. H. Holland. Adaptation in Natural and Artificial Systems. University of Michigan Press, Ann Arbor, 1975.

[34] D. E. Goldberg. Genetic Algorithms in Search Optimization and Machine Learning. AddisonWesley, Nova York, 1989.

[35] M. Mitchell. An Introduction to Genetic Algorithms. MIT Press, Cambridge, 1998.

[36] C. H. Papadimitriou and K. Steiglitz. Combinatorial Optimization - Algorithms and Complexity. Dover, 2 edition, 1998.

[37] S. Kirkpatrick, C. D. Gellat, and M. P. Vecchi. Optimization by simulated annealing. Science, 220:671-680, 1983.

[38] N. Metropolis, A. W. Rosenbluth, M. N. Rosenbluth, A. H. Teller, and E. Teller. Equation of state calculations by fast computing machine. Journal Chemical and Physical, 21:1087-1092, 1953.

[39] V. Cerny. Minimization of continuous functions by simulated annealing. Research Institute for Theoretical Physics, 1985.

[40] F. Glover. Heuristic for integer programming using surrogate contraints. Decision Sciences, 8:156-166, 1977.

[41] F. Glover. Future paths for integer programming and links to artificial intelligence. Computers and Operations Research, 13:533-549, 1986.

[42] R. Battiti and G. Tecchioli. The reactive tabu search. ORSA, Journal of Computing, 6:126-140, 1994.

[43] E. Burke, T. Curtois, M. Hyde, G. Kendall, G. Ochoa, S. Petrovic, J. A. V. Rodriguez, and M. Gendreau. Iterated local search vs. hyper-heuristics: Towards general-purpose search algorithms. In IEEE Congress on Evolutionary Computation (CEC), pages 1-8, July 2010 .

[44] J. Grobler, A. P. Engelbrecht, G. Kendall, and V. S. S. Yadavalli. Alternative hyper-heuristic strategies for multi-method global optimization. In IEEE Congress on Evolutionary Computation (CEC), pages 1-8, July 2010.

[45] W. A. Shewart. Economic control of Quality of Manufactured Product. Van Nostrand Reinhold Co., New York, 1931.

[46] E. L. Grant and R. S. Leavenworth. Statistical Quality Control. Tsinghua University Press, Beijing: China, seventh edition, 1999. 
[47] F. W. Breyfogle. Implementing Six Sigma: Smarter Solutions Using Statistical Methods. John Wiley \& Sons Ltda, New York, 1999.

[48] Q. L. Zhang. Metrics for meta-heuristic algorithm evaluation. In International Conference on Machine Learning and Cybernetics, volume 2, pages 1241-1244, November 2003.

[49] E. K. Burke, T. Curtois, M. Hyde, G. Kendall, G. Ochoa, S. Petrovic, and J. A. Vazquez-Rodriguez. Towards the decathlon challenge of search heuristics. In Genetic and Evolutionary Computation Conference (GECCO), pages 2205-2208, July 2009.

[50] K. A. De Jong. An analysis of the behavior of a class of genetic adaptive systems. PhD thesis, Department of computer and Communication Sciences, University of Michigan, Ann Arbor - Michigan, 1975.

[51] D. Whitley, K. Mathias, S. Rana, and J. Dzubera. Building better test functions. In Proceedings of the Sixth International Conference on Genetic Algorithms, pages 239-246, 1995.

[52] Z. Michalewicz. A hierarchy of evolution programs: an experimental study. In Evolutionary Computation, 1:51-76, 1993.

[53] J. D. Schaffer, R. Caruana, L. J. Eshelman, and R. Das. A study of control parameters affecting online performance of genetic algorithms for function optimization. In Proceedings of the 3rd International Conference on Genetic Algorithms, pages 51 - 60, 1989.

[54] D. H. Ackley. A connectionist machine for genetic hillclimbing. Kluwer Academic Publishers, Boston, 1987.

[55] L. A. Rastrigin. The convergence of the random search method in the extremal control of a many parameter system. Automation and Remote Control, 24(10):1337-1342, 1963.

[56] H. P. Schwefel. Numerical Optimization of Computer Models. PhD thesis, Birkhäuser Verlag, traduction by John Wiley and Sons in 1980, Basel, 1977.

[57] L. C. Dixon and G. P. Szego. The optimization problem: An introduction, Towards Global Optimization II. North Holland, New York, 1978.

[58] Z. Michalewicz. Genetic Algorithms + Data Structures = Evolution Programs. Springer-Verlag Berlin Heideberg, Berlin, 1996.

[59] D. Mackay. Good error-correcting codes based on very sparse matrices. IEEE Transactions on Information Theory, 45(2):399-431, March 1999.

[60] S. M. Alamouti. A simple transmit diversity technique for wireless communications. IEEE Journal on Selected Areas in Communications, 16(8):1451-1458, October 1998.

[61] Fernando Ciriaco. Otimização Multidimensional Baseada em Heurísticas Aplicada aos Sistemas de Comunicação Sem Fio. PhD thesis, Escola Politécnica da Universidade de São Paulo, March 2012. (in portuguese). 